Recibido: 25-05-2014

Aceptado: -20-08-2014

Palabras clave: población vegana, estado nutricional, composición corporal, distribución de grasa y perímetro

de cintura.

Keywords: vegan population, nutritional status, body composition, distribution of fat and waist circumference.

Milagros Jesús Saravia

Denegri

Colegio Villa María Miraflores milagros.saravia@gmail.

\section{Evaluación del estado nutricional, composición corporal y distribución de grasa en una población vegana*}

\section{Evaluation of nutritional status, body composition and distribution of fat in a vegan population}

\section{Milagros Jesús Saravia Denegri}

RESUMEN. El objetivo principal del estudio fue determinar el estado nutricional, la composición corporal y la distribución de grasa de la población vegana del Centro de Meditación Quan Yin, mediante un estudio descriptivo, no experimental de corte transversal, así como describir las características de la alimentación vegana. Esto permitirá profundizar más en el tema y servir de base para otras investigaciones.

La población estuvo constituida por la totalidad de los miembros del Centro de Meditación Quan Yin (44 adultos), de quienes se obtuvo el índice de masa corporal (IMC), porcentaje de grasa corporal (\% grasa), porcentaje de masa magra (\%masa magra) y perímetro de cintura. Se utilizó la prueba no paramétrica de la U de Mann-Whitney. El nivel de significancia fue de $\mathrm{p}=0.05$ con los respectivos grados de libertad. El análisis estadístico fue hecho con el programa SPSS.

Los resultados muestran una prevalencia de un IMC normal en el $77.3 \%$. En cuanto al $\%$ de grasa, predomina el sobrepeso leve (38.6\%); se encontró un \% de grasa óptimo en $23.3 \%$. Por otro lado, se observó que el grupo de \% de grasa corporal no óptimo (leve sobrepeso, sobrepeso y obeso) superó al de \% de grasa óptimo con un 72.73\%; el \% de masa magra se encontró aceptable en un 52.3\%. Se observó que un 72.8\% (32) no presenta riesgo cardiovascular, según perímetro de cintura.

Se concluye que las personas que ingieren una dieta vegana tienden a presentar un índice de masa corporal más bajo. Se confirmó que la dieta vegana está asociada con una menor tendencia a presentar riesgo cardiovascular y que planificadas correctamente son saludables, nutricionalmente adecuadas y ayudan a mantener un peso saludable.

ABSTRACT. The main objective of the study was to determine the nutritional status, body composition and distribution of fat in vegan population in Quan Yin Meditation Centre by a descriptive, non-experimental, cross-sectional study, as well as it describes the features of the vegan diet. This will go deeper into the issue and provide a basis for further research. The population consisted of all members of the Quan Yin Meditation Centre (44 adults), whom BMI, percent body fat, percent lean mass and waist circumference was obtained. Nonparametric test of Mann-Whitney was used. The level of significance was $\mathrm{p}=0.05$ with the respective degrees of freedom. Statistical analysis was done using SPSS. The results show a prevalence of $77.3 \%$ in normal BMI. As a percentage of fat, predominantly mild overweight (38.6\%); optimal fat percentage was found in $23.3 \%$. On the other hand it was observed that the group of body fat percentage than optimal (slightly overweight, overweight and obese) outperformed the optimal fat percentage with $72.73 \%$; percentage of lean mass was found acceptable in $52.3 \%$. It was found that $72.8 \%$ (32) no cardiovascular risk according to waist circumference. It was concluded that people who eat a vegan diet tend to have a lower rate of body mass. The percentage of fat vegans depend on the quality of their food (fried or natural food vs. processed). It was confirmed that the vegan diet is associated with a reduced tendency to cardiovascular risk. It has been found that properly planned vegan diets are healthful, nutritionally adequate and help maintain a healthy weight.

* Tesis para optar el Grado de Magíster en Nutrición y Dietética 


\section{Introducción}

$\mathbf{E}$

lserhumano, en el transcurso de su evolución, ha cambiado la percepción de la comida llevándola desde una mera herramienta de sobrevivencia hacia una instancia en la que se ven fuertemente involucradas ciertas prácticas, ritos, creencias y valores. Esto, ha llevado a una conjugación de aspectos sociales que implican el no consumo de carne animal y sus derivados. El veganismo es una tendencia alimentaria que excluye los alimentos y productos de origen animal. Esta tendencia ha ido en aumento en los últimos años demostrándose un auge del comercio enfocado a este público. Las personas que siguen esta dieta se someten a posibles deficiencias de macro y micronutrientes; debido a ello, buscan apoyo e información de diversas fuentes. Por otro lado, esta dieta se asocia a mayores beneficios para la salud por la composición de los lípidos y la mayor ingesta de fibra dietética. Así, la eliminación de la carne roja se ha asociado a una disminución del riesgo de desarrollar enfermedades cardiovasculares. La mayoría de las personas veganas refieren razones éticas, ecológicas, consideraciones de salud, o creencias religiosas como fundamentos para seguir este modelo alimentario. Finalmente, diversos estudios demuestran que estas personas presentan mejores estilos de vida. Ejemplos del creciente interés por el veganismo son el aumento de sitios webs que tratan el tema, la formación de agrupaciones con este interés en común, revistas y libros de cocina, la aparición de restaurantes veganos, comida rápida del mismo tipo, además de un gran aumento de la disponibilidad de productos aptos para veganos; en base a proteína vegetal y alimentos enriquecidos.

Esta investigación cobra importancia a nivel de salud, debido a la utilidad que pudiera tener los resultados de la evaluación obtenida en una población vegana, para la realización de posibles medidas alimentarias, tal es el caso de la dieta vegana, el consumo de alimentos y suplementos para satisfacer las necesidades de macro y micronutrientes de los veganos, debido a la exclusión total de alimentos de origen animal cuyos nutrientes pasan a ser críticos
Alétheia 2014, 2 (1) 165 a consecuencia de este tipo de dietas, tales como el calcio, hierro, zinc, vitamina B12, vitamina D, riboflavina y ácidos grasos omega-3 de cadena larga. Además, en el país, no se dispone de información sobre el estado nutricional, composición corporal y riesgo cardiovascular de la población vegana.

Esta investigación se justifica desde el punto de vista ético, debido a que los seres humanos, al consumir cualquier tipo de carne, están haciendo sufrir a sus co-habitantes, los animales, al ser asesinados para satisfacer el placer por la carne. Los animales, resulta obvio decirlo, experimentan dolor. Pueden sentirse solos, desesperados, tristes, sufren cuando se les priva de sus crías, notan el terror colectivo que se respira en los mataderos, y se apegan a la vida tanto como los seres humanos.

Un argumento que se aduce a favor de comer carne es que cuando se comen verduras también se está matando una vida. Es verdad que las plantas tienen conciencia, aunque muy rudimentaria, y experimentan sensaciones, pero no son en nada comparables con el sistema nervioso bien desarrollado de los animales. Los animales chillan, aúllan y se retuercen de sufrimiento, estas son manifestaciones palpables de que sienten el dolor en el mismo grado que los hombres.

El presente estudio reviste importancia porque cada vez hay más personas que optan por esta orientación alimentaria; siendo necesario y se debe detectar a tiempo posibles deficiencias de macro y micronutrientes.

El objetivo de la presente investigación fue evaluar el estado nutricional, la composición corporal y distribución de grasa corporal en una población vegana del centro de meditación Quan Yin.

Con relación a la bibliografía científica relacionada con el tema, se ha encontrado un estudio realizado en España (1) sobre la vitamina B12 en el vegetarianismo y los criterios para su diagnóstico, concluyendo que las dietas vegetarianas bien planificadas son nutricionalmente adecuadas y cubren los requerimientos necesarios para todas las etapas de la vida. Indicando también que, además del factor puramente dietético, existen otros muchos factores ajenos a este que pueden determinar la aparición de 
166 UNIFÉ - EPG

síntomas de deficiencia de vitamina B12 y que ponen en cuestión que una dieta vegetariana equilibrada y bien planificada, en un individuo en el que no se den estos otros factores, sea incapaz de aportar al organismo la cantidad de vitamina B12 necesaria para su correcto funcionamiento. La mayoría de estudios que se han realizado para valorar el problema del déficit de vitamina $\mathrm{B} 12$ en vegetarianos se han centrado en el problema del aporte dietético, pero han obviado otros factores que la mayor parte de las veces son determinantes para la aparición de los síntomas.

Otra investigación realizada en la India (2) sobre los Parámetros de densidad ósea, hematológica y bioquímica en vegetarianos y no vegetarianos, mostró que los lacto-ovo-vegetarianos tenían niveles más altos de glóbulos rojos y hematocritos que los no vegetarianos. Los veganos también tenían mayores valores de hematocrito que los no vegetarianos. Asimismo, se encontraron diferencias estadísticamente significativas entre los niveles plasmáticos de hierro en los grupos examinados. Los niveles de hierro eran más bajos en los no-vegetarianos que en los vegetarianos y los lacto-ovo-vegetarianos. Los no vegetarianos tenían niveles mucho más altos de colesterol, triglicéridos y colesterol LDL que los otros dos grupos, pero no se encontraron diferencias entre los mismos valores en los vegetarianos y los lacto-ovo-vegetarianos. Los investigadores también concluyen que una dieta vegetariana bien planificada y equilibrada, reduce los de riesgo de diversas enfermedades crónicas y carecniales..

Una investigación realizada en Chile (3) sobre la ingesta de alimentos de origen animal versus los de origen vegetal y riesgo cardiovascular, menciona que el aumento de la mortalidad cardiovascular en el último tiempo está fuertemente asociado a cambios en los hábitos de alimentación y concluye que la muerte por enfermedades cardiovasculares sigue aumentando de manera alarmante en el mundo, varias de sus causas como los hábitos de alimentación inadecuados, la baja actividad física y el consumo de tabaco son prevenibles, los resultados de esta revisión reafirman la importancia de emitir el mensaje a nivel poblacional de recomendar una disminución en el consumo de carnes rojas, principalmente procesadas, disminuir el consumo de grasas saturadas, trans, así como estimular el consumo de cereales integrales, frutas, vegetales y pescado para la disminución de la morbi-mortalidad global y cardiovascular.

Finalmente, un estudio realizado por Grigoletto y colaboradores (4) sobre la dieta vegetariana, niveles de colesterol y triglicéridos, concluyó que la dieta vegetariana se asocia a menores niveles de Triglicéridos, colesterol total y colesterol LDL en comparación con la dieta de omnívoros.

\section{Método}

\section{Tipo y diseño de la investigación}

Se realizó un estudio, cuantitativo, descriptivo y de corte transversal.

\section{Población y Muestra}

La población de estudio estuvo constituida por todos los miembros que asisten al Centro de Meditación Quan Yin; los cuales pasaron a conformar la muestra para el presente estudio. Esta muestra estuvo constituida por 44 personas de ambos sexos con edades comprendidas entre los 21 y 70 años.

\section{Métodos e instrumentos de recolección de datos}

Los datos fueron recogidos utilizando el método directo y con el siguiente procedimiento:

Evaluación nutricional.

a. Talla

Para medir la talla, se siguió procedimientos estandarizados.(5) Para ello, se pidió al sujeto colocarse en posición antropométrica, manteniendo los talones, los glúteos, la espalda y la región occipital en contacto con la pared (el plano vertical). Luego, se colocó un tope móvil sobre la cabeza del participante y se tomó la medida en centímetros. En el momento de la medida, el sujeto realizó una inspiración profunda 
para compensar el acortamiento de los discos intervertebrales. El instrumento utilizado fue la cinta antropométrica.

\section{b. Peso}

Cada voluntario se colocó encima de la balanza en la posición de atención antropométrica por un tiempo de 10 segundos los sujetos estuvieron completamente descalzos y sin metales ni joyería. Únicamente vistieron un short y un polo. La medida se obtuvo en kilogramos. (5)

c. Índice de masa corporal (IMC)

Se determinó el estado nutricional mediante el IMC Con los datos obtenidos anteriormente se aplicó la fórmula de Quetelet $\left(\mathrm{IMC}=\mathrm{P} / \mathrm{T}^{2}\right)(6)$. Finalmente se realizó la evaluación nutricional utilizando la clasificación de la OMS (7) registrando el resultado en el formato previamente diseñado.

\section{Composición corporal.}

Para medir la composición corporal, se utilizó el método de dos compartimentos, en donde el cuerpo se divide en: masa grasa (Fat) y masa libre de grasa (FFM). (8)

\section{a. Porcentaje de Grasa (\% de grasa)}

Para medir el \% de masa grasa, se utilizó una balanza de bioimpedancia eléctrica (BIA) marca TANITA, modelo BF-648W, con una capacidad de peso de 440 libras (220 kg.); para el efecto, se pidió a los voluntarios que vinieran los días de evaluación en la mañana temprano y en ayunas. Antes de la medición, tuvieron que evacuar la vejiga. También se les pidió que no ingirieran café, té o mates, ni que realizaran actividad física desde 12 horas antes de ser evaluados. En el caso de las mujeres, se pidió que separaran su día de evaluación lejos del período premenstrual para evitar la retención hidrica. (9)

Durante la medición, se pidió a los sujetos que subieran a la balanza con los pies descalzos, sin medias y se mantuvieran quietos por espacio de 15 segundos, al cabo del cual se registró la medida en el instrumento previamente diseñado.

Finalmente, se utilizó la tabla de Nieman para clasificar el porcentaje de grasa de los sujetos evaluados.

\section{b. Porcentaje de Masa Magra (\% masa magra)}

Este valor se obtuvo restando el $100 \%$ del peso - el $\%$ de grasa del sujeto, dando como resultado el \% de Masa Magra. Se consideró como valores aceptables una masa magra de 75 por ciento en las mujeres y de 80 por ciento en los hombres. (11)

\section{Distribución de Grasa en el Perímetro de Cintura}

Para conocer la distribución de grasa en la zona abdominal, el participante se colocó en posición antropométrica. La medición se realizó a la altura del ombligo con el abdomen descubierto, sin oprimir el tejido. Se utilizó la cinta antropométrica inextensible, flexible, metálica, de anchura inferior a $7 \mathrm{~mm}$ con una precisión de $1,1 \mathrm{~mm}$. El resultado se obtuvo en centímetros. (12). Finalmente, se utilizó la tabla de la Asociación Americana de Diabetes para clasificar el riesgo cardiovascular, según el perímetro de cintura de los sujetos evaluados. (13) Luego, se registró el resultado en el formato previamente diseñado.

\section{Características demográficas.}

Para conocer las características demográficas, se entrevistó a cada uno de los 44 miembros del Centro de Meditación Quan Yin, respecto a su procedencia, datos generales así como las razones que los motivó a convertirse en veganos, recolectando la información en un instrumento previamente validado.

\section{Resultados}

Los siguientes resultados fueron obtenidos, durante el mes de noviembre del año 2012, en la población vegana del Centro de Meditación Quan Yin, ubicado en el distrito de La Molina. Actualmente, se cuenta con 44 miembros veganos, de los cuales el 50\% perteneció al género masculino y el 50\% al femenino.

\section{Estado Nutricional}

En la tabla 1, se muestran los resultados del estado nutricional de la población en estudio. Observamos que el 77.3\% de la población estudiada presentó un estado nutricional normal; el 18.2\% de 
168 UNIFÉ - EPG

la población presentó sobrepeso; el 4.5\% presentó obesidad de tipo I.

Efectivamente, existen diferencias significativas entre el grupo de sujetos con estado nutricional normal y los otros grupos.

Por lo tanto, se verificó la hipótesis: el estado nutricional de la población vegana en estudio se encuentra dentro de los parámetros normales (Fig. 1). Tabla 1

Análisis de datos referidos al estado nutricional del vegano, usando la Chi cuadrada

\begin{tabular}{cccccc}
\hline Normal & Sobrepeso & Obeso 1 & Total & $\begin{array}{c}\text { Chi } \\
\text { cuadrada }\end{array}$ & gl \\
\hline 34 & 8 & 2 & 44 & $39.455^{*}$ & 2 \\
$77.3 \%$ & $18.2 \%$ & $4.5 \%$ & $100 \%$ & & \\
\hline${ }^{*}<0.05$ & & & & &
\end{tabular}

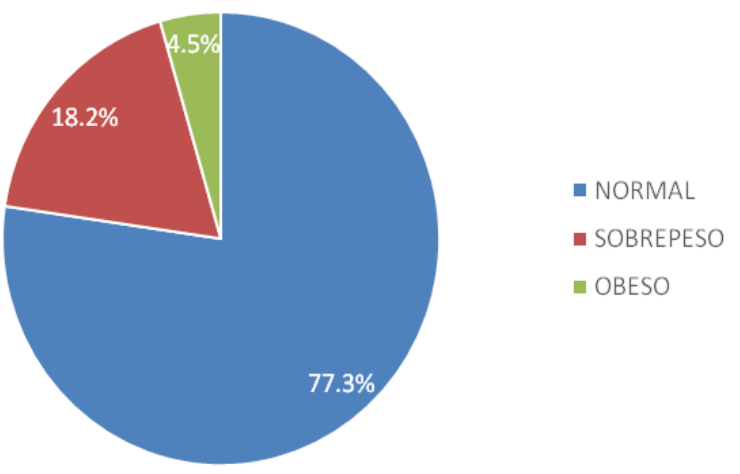

Figura 1. Estado nutricional de la población vegana en estudio.

\section{Composición Corporal}

En la tabla 2, se muestran los resultados referentes a la composición corporal, en cuanto al \% de grasa, de la población en estudio. Se observa en esta tabla que el $27.7 \%$ de la población estudiada presentó un $\%$ de masa grasa óptimo; el 38.6\% presentó un $\%$ de masa grasa de leve sobrepeso; el $20.5 \%$ de la población presentó un $\%$ de masa grasa de sobrepeso, y el $13.6 \%$ de la población presentó un \% de masa grasa de obeso. Los resultados indicaron que no existen diferencias significativas en cuanto al \% de masa grasa óptimo y los otros grupos. No se verificó la hipótesis planteada: la composición corporal de la población vegana en estudio se encuentra dentro de los parámetros normales (Fig. 2).
Tabla 2

Análisis de datos referidos a la composición corporal, en cuanto al \% de grasa del vegano, usando la Chi cuadrada.

\begin{tabular}{ccccccc}
\hline Optimo & $\begin{array}{c}\text { Leve } \\
\text { Sobrepeso }\end{array}$ & sobrepeso & obeso & Total & $\begin{array}{l}\text { Chi } \\
\text { cuadrada }\end{array}$ & gl \\
\hline 12 & 17 & 9 & 6 & $39.455^{*}$ & 13.92 & 3 \\
$27.7 \%$ & $38.6 \%$ & $20.5 \%$ & $13.6 \%$ & $100 \%$ & & \\
\hline$*_{\mathrm{p}}<0.05$ & & & & & &
\end{tabular}

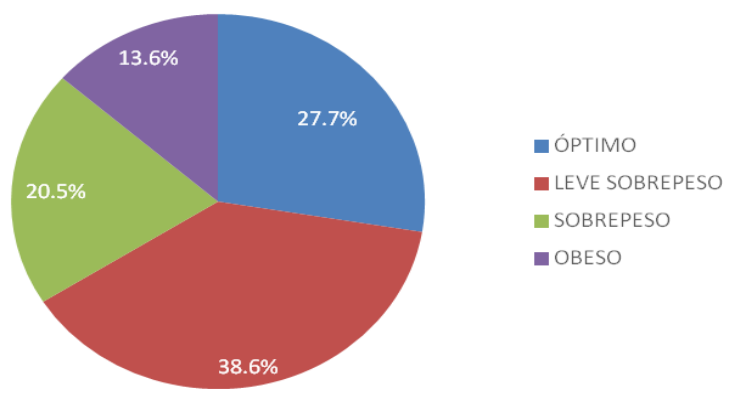

Figura 2. Composición corporal, en cuanto al \% de grasa, de la población vegana en estudio.

Asimismo, en la tabla 3 se muestran los resultados referentes a la composición corporal, en cuanto al \% masa grasa de la población en estudio reagrupados en dos categorías: porcentaje de masa grasa "óptimo" y porcentaje de masa grasa "no óptimo". Se observa que se han reagrupado los datos, en el sentido de que la categoría "no óptimos" supera a los "óptimos". Al respecto, observamos que el 27.7\% de la población presentó un \% de masa grasa dentro del rango óptimo, y el $72.73 \%$ presentó un \% de masa grasa no óptimo. Por lo tanto, los resultados indicaron que sí existen diferencias significativas en cuanto al \% de masa grasa; por lo tanto, se rechaza la hipótesis planteada: la composición corporal respecto al \% de masa grasa de la población vegana se encuentra dentro de los parámetros normales (Fig. 3).

Tabla 3

Análisis de datos referido a la composición corporal, en cuanto al $\%$ de grasa del vegano, usando la Chi cuadrada.

\begin{tabular}{ccccc}
\hline Óptimo & $\begin{array}{c}\text { No } \\
\text { Óptimos }\end{array}$ & Total & $\begin{array}{c}\text { Chi } \\
\text { cuadrada }\end{array}$ & gl \\
\hline 12 & 32 & 44 & & \\
$27.27 \%$ & $72.73 \%$ & $100 \%$ & $9.091 *$ & 1 \\
\hline${ }^{\mathrm{p}}<0.05$ & & & &
\end{tabular}




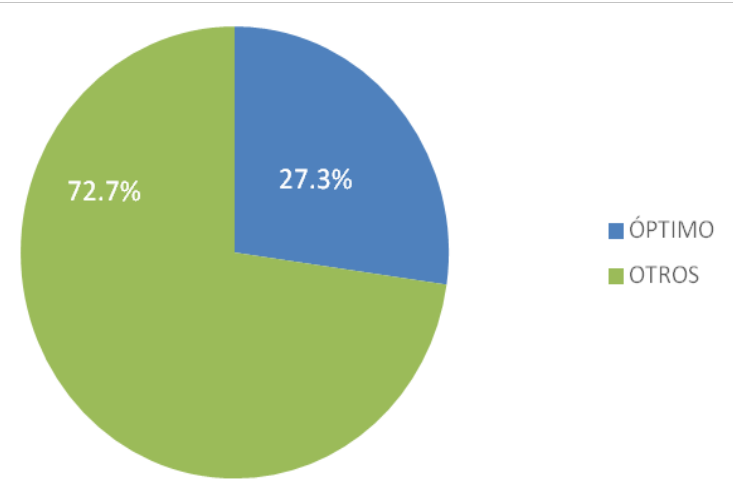

Figura 3. Composición corporal, en cuanto al \% de grasa, de la población vegana en estudio.

En la tabla 4 y figura 4, se muestran los resultados referentes a la composición corporal, en cuanto al \% de masa magra de la población en estudio. En esta tabla, se observa que el $52.3 \%$ de la población presentó un $\%$ de masa magra dentro del rango "aceptable" y el 47.7\% de la población presentó un $\%$ de masa magra "no aceptable". Por lo tanto, los resultados indicaron que no existen diferencias significativas en cuanto al \% de masa magra en la población en estudio.

Tabla 4

Análisis de datos referidos a la composición corporal, en cuanto al \% de masa magra, del vegano usando la Chi cuadrada.

\begin{tabular}{ccccc}
\hline Aceptable & $\begin{array}{c}\text { No } \\
\text { Aceptable }\end{array}$ & Total & $\begin{array}{c}\text { Chi } \\
\text { cuadrada }\end{array}$ & gl \\
23 & 21 & 44 & & \\
$52.3 \%$ & $47.7 \%$ & $100 \%$ & 0.091 & 1 \\
\hline
\end{tabular}

$*_{\mathrm{p}}<0.05$

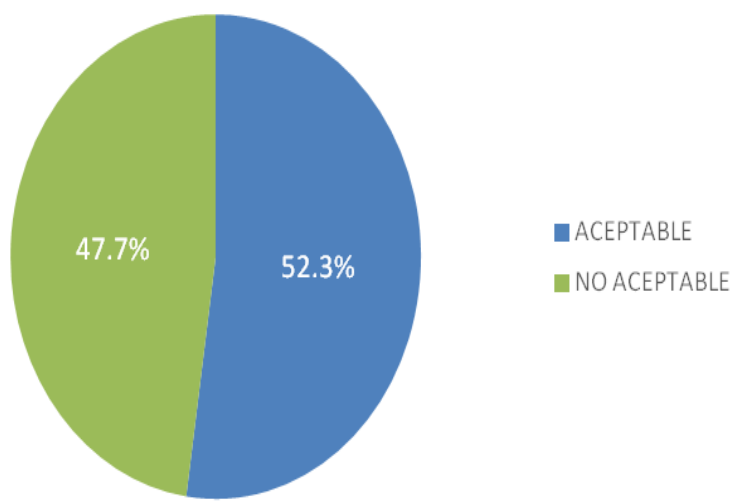

Figura 4. Composición Corporal, en cuanto al \% de masa magra de la población vegana en estudio.

\section{Distribución de grasa corporal}

En la tabla 5, se muestran los resultados referentes a la distribución de grasa según el perímetro de cintura de la población en estudio y según su género. En esta tabla, se observa que el promedio de circunferencia de cintura en mujeres fue de $81 \mathrm{~cm}$ y en varones de $88 \mathrm{~cm}$. Por lo tanto, los veganos en estudio presentaron un promedio de cintura dentro del rango saludable.

Tabla 5

Análisis de datos referidos al perímetro de cintura del vegano, según su género

\begin{tabular}{ccc}
\hline Género & Masculino & Femenino \\
\hline Promedio & $81 \mathrm{~cm}$ & $88 \mathrm{~cm}$ \\
Perímetro Cintura & & \\
\hline
\end{tabular}

Riesgo Cardiovascular

En la tabla 6, se muestran los resultados referentes al riesgo cardiovascular de la población en estudio según su perímetro de cintura. Se observa que el $72.8 \%$ de la población no presentó riesgo cardiovascular; el $11.3 \%$ presentó riesgo elevado para su salud y el $15.90 \%$ de la población presentó riesgo muy elevado. Por lo tanto, los resultados indicaron que sí existen diferencias significativas entre los grupos que no presentan riesgo cardiovascular y los otros grupos; por lo tanto, se acepta la hipótesis: la población en estudio no presenta riesgo cardiovascular (Fig. 6).

Tabla 6

Análisis de datos referidos al riesgo cardiovascular considerando

\begin{tabular}{cccccc} 
el perimetro de cintura del vegano, usando la Chi cuadrada \\
\hline Normal & $\begin{array}{c}\text { Riesgo } \\
\text { elevado } \\
\text { de salud }\end{array}$ & $\begin{array}{c}\text { Riesgo } \\
\text { muy } \\
\text { elevado }\end{array}$ & Total & $\begin{array}{c}\text { Chi } \\
\text { cuadrada }\end{array}$ & Gl \\
\hline 32 & 5 & 7 & 44 & & \\
$72.8 \%$ & $11.3 \%$ & $15.90 \%$ & $100 \%$ & $30.864 *$ & 3 \\
\hline
\end{tabular}

${ }^{*} \mathrm{p}<0.05$ 


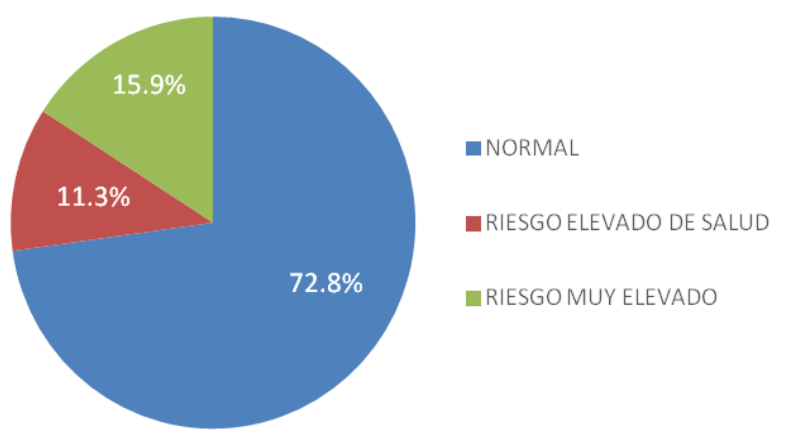

Figura 6. Riesgo Cardiovascular de la población vegana en estudio.

\section{Discusión de Resultados}

\section{Estado nutricional}

Respecto a los resultados del estado nutricional de la población en estudio, se observó un 77.3\% dentro de los parámetros normales que, comparado con lo publicado por la American Dietetic Association (Asociación Americana de Dietética), en julio de 2009, los individuos que son vegetarianos desde nacimiento, de adultos presentan una estatura, un peso y un IMC similares a los de aquellos que se hicieron vegetarianos en etapas posteriores de su vida, lo cuál sugiere que durante la infancia y la niñez las dietas vegetarianas bien planificadas no afectan a la estatura o al peso finales del adulto y que son apropiadas para todas las etapas del ciclo vital, incluyendo el embarazo y la lactancia. (14)

Asimismo, hay distintas investigaciones (15) que concuerdan con el resultado obtenido. Por ejemplo, entre los adventistas quienes en promedio siguen una dieta sin carne, los patrones de alimentación vegetariana han sido asociados con un menor IMC, el cuál se incrementa a medida que crece la frecuencia de consumo de carne, tanto en hombres como en mujeres.(16) Otro estudio realizado por la Universidad de Oxford sobre obesidad y vegetarianismo, observó que los valores de IMC fueron superiores en los no vegetarianos en comparación con las personas vegetarianas en todos los grupos de edad y en ambos sexos. (17) Asimismo, en un estudio transversal realizado en 37.875 adultos que consumían una dieta carnívora, presentaron los valores más altos de IMC en comparación a los veganos (18). Comparando estos estudios con el nuestro, se puede comprobar también que los veganos tienen la tendencia de presentar un IMC menor. Asimismo, un estudio transversal realizado en Inglaterra, mostró que las personas que se hicieron vegetarianas de adultas no diferían en IMC o peso corporal respecto a aquellas que eran vegetarianas de por vida. (19) Finalmente, se puede mencionar que se ha comprobado que una dieta vegetariana baja en grasas es más efectiva para la pérdida de peso a largo plazo en mujeres postmenopáusicas que una dieta más convencional (20).

\section{Composición Corporal}

Porcentaje de masa grasa

Al analizar la composición corporal de la población vegana en cuanto a su \% de masa grasa, se encontró que no hay diferencias significativas, debido a que el grupo con \% de masa grasa óptimo constituyó solo el $27.7 \%$ de la muestra; sin embargo, reagrupando a la población en dos grupo: \% de masa grasa "óptima" fue representado en un $27.27 \%$ de la población, en comparación del grupo considerado con masa grasa "no óptima" que constituyó el $72.73 \%$. Este resultado estaría indicando que existen veganos que presentan porcentajes de grasa elevados, resultado que contradice los estudios mencionados anteriormente, lo que podría originar problemas de sobrepeso u obesidad. Esto podría deberse al elevado consumo de carbohidratos y frituras observado en la población en estudio. Serían los llamados "veganos chatarreros". El hecho de que la población estudiada sea vegana no quiere decir que su alimentación sea la más óptima, o que siempre este consumiendo vegetales y frutas. Sobre todo si se volvieron veganos por un tema filosófico o ecológico y no tanto por motivos de salud.

$\mathrm{Al}$ respecto, la revista Vitónica sobre alimentación, deporte y salud (21), indica que aunque muchos se inician en la dieta vegetariana con la idea de perder peso, lo cual es producto de la falsa creencia 
de que comiendo alimentos de origen vegetal es más fácil adelgazar, en realidad, es muy complejo revertir un sobrepeso siendo vegetariano y además, la relación vegetarianismo y sobrepeso cada vez es más frecuente. Los vegetarianos pueden tener sobrepeso debido al alto consumo de hidratos de carbono derivados de cereales, legumbres, frutas y verduras y también, debido al consumo de aceites vegetales. Asimismo, la baja ingesta de proteínas que sacian con facilidad y demandan un gran gasto calórico para digerirse, pueden ser razones suficientes para asegurar la relación entre sobrepeso y vegetarianismo.

Por el contrario, en el caso de los ovo- lacto vegetarianos la cuestión cambia a favor de revertir el sobrepeso, pues incluyen lácteos desnatados y clara de huevo, proteínas que revisten saciedad.; por otro lado, el vegetariano estricto quien consume proteínas derivadas de las legumbres y frutos secos, resulta más complejo reducir las fuentes de energía que permitan remover grasas. La opción que queda para revertir sobrepeso en vegetarianos es reducir el tamaño de las porciones, aliarse de las legumbres que tienen baja densidad calórica y la práctica de actividad física.

\section{Porcentaje de masa magra}

Al analizar la composición corporal de la población vegana en estudio, en relación a su $\%$ de masa magra, no se encontraron diferencias significativas entre los grupos calificados con masa magra "aceptable" en relación al grupo con masa magra "no aceptable". Se puede observar, entonces, que no necesariamente la mayoría de los veganos presentan un \% de masa magra dentro del rango normal. Esto podría deberse al elevado consumo de proteínas de origen vegetal y la falta de actividad física.

\section{Riesgo Cardiovascular}

Con relación al riesgo cardiovascular se observó que la población en estudio no presenta riesgo cardiovascular según su perímetro de cintura, comparado con otros autores(3) quienes concluyen que el consumo de cereales integrales, frutas y vegetales que habitualmente consumen los veganos son beneficiosos para su salud y ayudan en la prevención de las enfermedades cardiovasculares.

De la misma manera, la Sociedad Latinoamericana de Cardiología Intervencionista, (22) se demostró que los vegetarianos tienen un riesgo de sufrir enfermedades vasculares un 32\% menor. La investigación en 45.000 hombres y mujeres señaló que también era menor el riesgo de diabetes y bajo índice de masa corporal. Los resultados muestran el importante papel de la dieta en la prevención de enfermedades cardíacas.

La disminución del riesgo cardiovascular para los vegetarianos también se ha demostrado en el estudio de la Universidad de Oxford (20) , realizado 44.561 sujetos de ambos sexos; se obtuvo como resultado que el $34 \%$ consumía una dieta vegetariana, y luego de un seguimiento de 11 años, se determinó que los vegetarianos tenían un riesgo cardiovascular $32 \%$ menor de sufrir internaciones por infarto de miocardio. Esto fue demostrado de manera independiente a los factores de riesgo como el sexo, la edad, la obesidad u otros. Este resultado "se debe probablemente a diferencias en el colesterol y la presión arterial".

Finalmente, deben recalcarse que los vegetarianos no solo se beneficiaban del impacto positivo de registrar un menor índice de masa corporal, sino que también se reduce en un 28\% el riesgo de padecer enfermedades cardiovasculares.

\section{Conclusiones}

1. El $77 \%$ de la población en estudio, según el IMC, presentó un estado nutricional normal; por lo tanto, se verificó la hipótesis de que el estado nutricional según sexo de la población vegana se encuentra dentro del rango normal. $(\mathrm{p}<0.05)$.

2. El $27.27 \%$ de los veganos presentaban rangos normales de masa grasa, y el $72.73 \%$ fue calificado con un porcentaje de masa grasa "no óptimo". Por lo tanto, se rechazó la hipótesis planteada, asimismo se observó de que existen diferencias significativas según género $(\mathrm{p}<0.05)$. 
172 UNIFÉ - EPG

3. El $52.3 \%$ de la población estudiada presentó un porcentaje de masa magra dentro del rango normal, calificada como "aceptable". Por lo tanto, no existen diferencias significativas en la composición según masa magra de esta población, y tampoco existen diferencias significativas según sexo.

4. Respecto a la distribución de grasa abdominal, se observó que el promedio de circunferencia de cintura en mujeres fue de $81 \mathrm{~cm}$. y en varones de $88 \mathrm{~cm}$. Asimismo, el $72.8 \%$ de la población vegana no presentó riesgo cardiovascular. $(\mathrm{p}<0.05)$. En cuanto a género, no se observó diferencias significativas.

5. Respecto a los datos demográficos, se observó que el $44 \%$ de la población vegana procede de Lima y el $43 \%$ tiene edades entre 41 a 60 años, el $73 \%$ viene practicando el veganismo durante un período de 6 a 10 años y que el 91\% de la población estudiada indicó que los motivos principales para adoptar esta práctica fueron éticos y ecológicos.

\section{Referencias}

1. Pueyo-Rodrigo Ma Te, Vitamina B12 en el Vegetarianismo. Criterios para su diagnóstico (en línea.) Rev Med Nat, Zaragoza, España, 2007; Vol1- N 2: 120- 130.

2. Sambol SZ, Štimac D, Orlić ŽC, Guina T, Parámetros de densidad ósea, hematológica, y bioquímica en vegetarianos y no vegetarianos West Indian med. J. [en línea]. 2009 Dic [03 de jul de 2012] vol.58 no.6 Disponible en: http:// caribbean.scielo.org/scielo.php?script $=$ sci_ arttext\&pid=S0043-31442009000600004\&lng $=\mathrm{e}$ s\&nrm $=$ iso

3. Pino Ángela, Cediel Gustavo, Hirsch Sandra, Ingesta de alimentos de origen animal versus origen vegetal y riesgo cardiovascular, Rev. chil. Nutr [en línea] 2009 Set [accesado 02 de jul. 2012]; vol.36 no.3. Disponible en: http://www. scielo.cl/scielo.php?script=sci_arttext\&pid $=\mathrm{S} 0717-75182009000300003$

4. Grigoletto De Biase S, Carrocha Fernandes S F,
Gianini R J, Jarcia Duarte J L. Dieta vegetariana y nivelesdecolesteroly triglicéridos. Arq. Bras. Cardiol [en línea] 2007 ene [accesado 29 de jun. 2012]; vol.88 no.1. Disponible en: http://www.scielo.br/ scielo.php?script $=$ sci_arttext\&pid=S0066-782X2 007000100006\&lng $=\mathrm{pt \& nrm}=$ iso\&tlng $=\mathrm{pt}$

5. García-Molina MR, Estimación de la Composición Corporal: Medidas Antropométricas. efdeportes. com Rev Dig [en línea]. Buenos Aires: Año 14 $\mathrm{N}^{\circ}$ 140, 2010 ene [accesado 03 de diciembre 2012]. Disponible en: http://www.efdeportes. com/efd140/composicion-corporal-medidasantropometricas.htm

6. ClínicaMédicaInternacionalparaReduccióndePeso [en línea].85 México: clinicamedicainternacional. com; 2004 [accesado 02 de dic 2012]. Indice de Masa Corporal [2 pantallas]. Disponible en: http://www.clinicamedicainternacional.com/ indice_masa_corporal.html

7. World Health Organization [en línea] who.int [accesado 30 may 2014]. BMI Classification [2 pantallas]. Disponible en: http://apps.who.int/ bmi/index.jsp?introPage $=$ intro_3.html

8. Archivos de Medicina del Deporte [en línea] femede.es; 2004 [accesado 02 de jun 2014] Vol XXI - $\mathrm{N}^{\circ}$ 104. Métodos de evaluación de la composición corporal: Evidencias Actuales. [4 pantallas] Disponible en: http://femede.es/ documentos/Rev_tendencias\%20I_535_104.pdf

9. Vitónica Alimentación, Deporte y Salud [en línea]. Gottau G. vitonica.com; 21 de julio de 2010 [accesado 03 de dic 2012]. Para Tener en Cuenta al Medir Grasa con Impedancia [3 pantallas]. Disponible en: http://www.vitonica.com/ profesional/para-tener-en-cuenta-al-momentode-medir-grasa-con-impedancia

10. Fórmulas y Tablas [en línea] scribd.com [accesado 02 de jun.]. Fórmulas de antropometría. [2 pantallas]. Disponible en: http://es.scribd.com/ doc/95192995/Formulas-y-Tablas-Si

11. La masa magra o masa grasa [en línea]. Joiagroup. com; [accesado 04 de Dic. 2012]. Disponible en: http://joiagroup.com/contenidos/4/article_701. htm 
12. García- Molina MR, Estimación de la Composición Corporal:Medidas Antropométricas. efdeportes.com Rev Dig [en línea]. Buenos Aires: Año $14 \mathrm{~N}^{\circ}$ 140, 2010 ene [accesado 03 de diciembre 2012]. Disponible en: http://www. efdeportes.com/efd140/composicion-corporalmedidas-antropometricas.htm

13. Cicloba [en línea] ciclobr.com; feb. 2008, [accesado 01 de jun. 2014]. Perímetro de la cintura medida para conocer el riesgo cardiovascular y Alzheimer. [2 pantallas]. Disponible en: http://www.ciclobr.com/ cintura.html

14. Unionvegetariana.org [en línea]. Argentina; unionvegetariana.org [accesado 02 de ene 2014.] Obesidad y Vegetarianismo [2 pantallas]. Disponible en: http://www.elenciclopedista.com.ar/obesidad$\mathrm{y}$-vegetarianismo/

15. Vitonica.com [en línea]. vitonica.com; 2011 oct 25 [accesado 02 de ene 2014.] Vegetarianos y Sobrepeso: Una relación posible [2 pantallas.] Disponible en: http://www.vitonica.com/prevencion/ vegetarianos-y-sobrepeso-una-relacion-posible

16. P.N. Appleby, M. Thorogood, J.I. Mann and T.J. Key, The Oxford Vegetarian Study: An overview, Am J Clin Nutr [en línea] 1999 Sept [accesado 16 feb 2014] pp. 525S-531S. Disponible en: http:/ /ajcn.nutrition. org/content $/ 70 / 3 / 525$ s.full

17. E.A. Spencer, P.N. Appleby, G.K. Davey and T.J. Key, Diet and body-mass index in 38000 EPIC-Oxford meat-eaters, fish-eaters, vegetarians, and vegans, Int J Obes Relat Metab Disord [en línea] 2003 [accesado 16 feb 2014] pp. 728-734. Disponible en: http:/ /veg. ca/wp-content/uploads/2013/01/bodymassindex. pdf

18. M. Rosell, P. Appleby, E. Spencer and T. Key, Weight gain over 5 years in 21,966 meat-eating, fish-eating, vegetarian, and vegan men and women in EPICOxford, Int J Obesity [en línea] 2006 [accesado 16 feb 2014] pp. 1389-1396. Disponible en: http:// www.readcube.com/articles/10.1038/sj.ijo.0803305

19. Braithwaite, H.S. Fraser, N. Modeste, H. Broome and R. King, Obesity, diabetes, hypertension, and vegetarian status among Seventh-day Adventists in Barbados: Preliminary results, Eth Dis[en línea] 2003 [accesado 16 feb 2014] pp. 34-39. Disponible
Alétheia 2014, 2 (1) 173

en: http://www.grep-aedn.es/documentos/ vegetariandiets.pdf

20. Página12 [en línea] Argentina. pagina12.com; 31 ene 2013[accesado 16 feb 2014]. Sociedad Dieta Vegetariana y Enfermedades Cardiacas. Un Riesgo Disminuido. Disponible en: http://www.pagina12. com.ar/diario/sociedad/3-212955-2013-01-31. html

21. Vitónica solaci.org [en línea]. solaci.org; [accesado 02 de ene 2014.] Los Vegetarianos tienen menor riesgo cardiovascular [2 pantallas.] Disponible en: http:/ / solaci.org/es/vegetarianos_tienen_menor_riesgo_ cardiovascular.php

22. G.E. Fraser, Associations between diet and cancer, ischemic heart disease, and all-cause mortality in nonHispanic white California Seventh-day Adventists, Am J Clin Nutr [en línea] 1999 Sept [accesado 16 feb 2014]; pp. 532S-538S. Disponible en: http:// ajcn.nutrition.org/content/70/3/532s.full 